\title{
INFLUÊNCIA DO ENRIQUECIMENTO AMBIENTAL NOS PADRÕES DE COMPORTAMENTOS SOCIAIS E ANORMAIS DE CABRAS EM CONFINAMENTO
}

\author{
(influence of environmental enrichment in patterns of social behaviour and \\ abnormal of goats in containment)
}

\author{
Ana Paula Guedes Oliveira, Weliton Menário Costa, Willian Moreira da Costa, \\ Rafael de Almeida Nunes, Natalia Caroliny da Silva Dias, Aparecida de Fátima \\ Madella-Oliveira ${ }^{1}$ \\ Correspondência: amadella@ifes.edu.br
}

\begin{abstract}
RESUMO: A partir do processo de domesticação as cabras foram retiradas do seu ambiente natural e inseridas em um ambiente empobrecido em estímulos. O problema é que, em função do confinamento inadequado, os animais deixam de realizar comportamentos típicos da espécie e passam a desenvolver comportamentos anormais. Na tentativa de minimizar esses comportamentos, têm-se utilizado a técnica denominada enriquecimento ambiental. $O$ objetivo do trabalho foi identificar, analisar e descrever os comportamentos sociais agonísticos e anormais de cabras confinadas com e sem o uso de enriquecimentos ambientais, relacionando-os ao ganho de peso. Para o desenvolvimento do trabalho, utilizaram-se 20 cabras mestiças (Saanen $x$ Boer), igualmente divididas em duas baias, sendo a baia controle (Trat 1) tratada conforme a rotina do setor e a baia de enriquecimento (Trat 2) submetida à técnica de enriquecimento ambiental. Realizaram-se observações do tipo focal, perfazendo um total de 216 horas. Verificou-se que as médias de frequência dos comportamentos em cada tratamento diferiram entre si, sendo que na baia de enriquecimento ambiental os animais exibiram menor frequência de comportamentos sociais agonísticos e anormais em relação à baia controle. Conclui-se que o enriquecimento ambiental influenciou de forma positiva no comportamento anormal e social das cabras estudadas.
\end{abstract}

Palavras-chave: bem-estar; caprino; domesticação; peso

\begin{abstract}
Throughout the history of the animal domestication process, goats were removed from their natural environment and they were placed in an environment with impoverished stimuli. The problem is that because of the confinement, the animals fail to display behaviors typical of the species and they begin to develop abnormal behaviors. In an attempt to minimize these behaviors, it has become common to use a technique named environmental enrichment. The aim was to identify, analyze and describe the agonistic and abnormal social behaviors of confined goats with and without using environmental enrichments, relating them to weight gain. For study development, w e used 20 crossbred goats (Boer $x$ Saanen), equally divided into two pens, where a group control was treated according to the normal routine of the farm (Trat 1) and another one, the enrichment group, was subjected to the technique of environmental enrichment (Trat 2). The two groups were then observed, making a total of 216 hours. It was found that the mean frequency of behaviours for each treatment differ, and that the group with the environmental enrichment animals exhibited lower frequency of agonistic social and abnormal behavior than the control group. It is therefore concluded that environmental enrichment positively influenced the abnormal and social behavior in the goats studied.
\end{abstract}

Key Words: domesticated; goats; weight; welfare 
se uma técnica denominada

\section{INTRODUÇÃO}

\section{Evidências}

arqueológicas mostram que a cabra doméstica (Capra hircus) descendente da cabra selvagem (Capra aegagrus) ou Benzoar, foi domesticada no Crescente Fértil, região do Oriente Médio, na Ásia à aproximadamente dez mil anos (Zeder e Hesse, 2000).

No Brasil a entrada desses animais deve-se aos colonizadores e apresenta-se dividida em duas fases, sendo a primeira do século XVI ao XVIII com a introdução de animais não padronizados e a segunda a partir do século XIX com a chegada das raças modernas que se deu em 1975 quando o segundo Plano Nacional de Desenvolvimento proibiu a importação de produtos provenientes desses animais, permitindo o ingresso, dentre outras, da raça Saanen (Machado Filho e Hotzel, 2000).

Desde então o cultivo dessa raça tem aumentado significativamente, em função da alta produtividade de leite. É válido ressaltar que 0 ambiente de cativeiro é diferente daquele no qual a espécie evoluiu (Sgai, 2007). Este ambiente pode afetar 0 bem-estar desses animais que por falta de estímulo físico e mental, apresentam agressividade, automutilação, hipersexualidade, estereotipia, apatia e depressão, podendo até mesmo levar à morte (Burn et. al., 2010; Hammerschmidt, 2012), além de comportamentos como roer a baia (Malafaia et. al., 2011).

Contudo, determinar um ambiente ideal é uma tarefa complicada, pois para tanto, deve-se levar em consideração as necessidades comportamentais do animal e estas ainda não são bem definidas (Yeates, 2011).

Para melhorar 0 ambiente e consequentemente, o bem-estar, utiliza- enriquecimento ambiental que consiste em modificações no ambiente físico ou social do animal combinando conhecimento prévio do habitat e do comportamento natural ou típico da espécie, visando sua melhoria ou prevalência, bem como a redução dos níveis de estresse, melhoria na saúde e desempenho reprodutivo (Pizzuto et. al., 2009; Campos et. al., 2010; Mendes et. al., 2011).

O estudo do comportamento animal possibilita ao produtor conhecer os hábitos dos animais $\mathrm{e}$, a partir de alterações nos padrões comportamentais, conduzir adequadamente as práticas de manejo, visando à redução de custos e o aumento na produção e produtividade dos animais (Ribeiro et. al., 2009; Silva, 2011).

Neste contexto, o objetivo do trabalho foi identificar, analisar e descrever os comportamentos sociais agonísticos e anormais de cabras confinadas com e sem o uso de enriquecimentos ambientais, relacionando-os ao ganho de peso.

\section{METODOLOGIA}

O trabalho foi desenvolvido no setor de caprinocultura do Instituto Federal de Educação, Ciência e Tecnologia do Espírito Santo - Campus de Alegre, situado às coordenadas

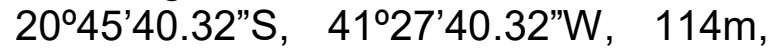
distrito de Rive, Alegre, região Sul do Espírito Santo, Brasil, entre os meses de março e maio de 2012.

Foram utilizadas 20 cabras mestiças (Saanen x Boer), com oito meses de idade. Os animais eram alimentadas, em cocho, duas vezes ao dia, de manhã (7h) e à tarde (15h). As forragens ofertadas pelo setor foram capim-elefante picado, cana-de-açúcar picada e capim tífton na forma de feno. 

anormais de cabras em confinamento

Também foi ministrado um concentrado composto de $22 \%$ de proteína bruta, $2,3 \%$ de extrato etéreo, $4,30 \%$ de fibra bruta, $1,20 \%$ de cálcio, $0,30 \%$ de fósforo, $71,50 \%$ de NDT e $18 \mathrm{mg}$ de promotor de crescimento adicionado à forragem na proporção de $2 \%$ do peso vivo. O consumo de alimentos, de sal mineral e de água pelos animais foi ad libitum.

As fêmeas foram pesadas duas vezes, sendo uma no início e outra no final do experimento.

O método utilizado para observação dos comportamentos foi o amostral focal.

As cabras foram mantidas em duas baias, de dimensão $8 \times 5 \mathrm{~m}$, sendo dez cabras por baia, respeitando sua disposição original no setor e, para facilitar a identificação pelo observador, elas foram marcadas com spray atóxico em regiões do dorso e, fitas de cetim de colorações variadas envoltas no pescoço.

Primeiramente, construiu-se um etograma com os principais padrões comportamentais sociais agonísticos e anormais. Foram realizadas três horas de observações diárias, intercaladas entre as duas baias, sendo $1 \mathrm{~h} 30 \mathrm{~min}$ por turno (matutino e vespertino), perfazendo 72 horas.

Os animais foram redistribuídos em duas novas baias de dimensões $8 \times 5 \mathrm{~m}$, com 10 animais cada, selecionados de forma aleatória através de um sorteio, procedendo-se com diferentes tratamentos em cada uma. $\mathrm{Na}$ baia controle (Trat 1) os animais não foram submetidos a nenhuma intervenção, enquanto a baia de enriquecimento (Trat 2) foi incrementada com diferentes tipos de enriquecimentos ambientais (Tabela 1), tais como música, brinquedos e alimentação diferenciada (Figura 1), intercalados entre os dias da semana.
Tabela 1 - Tipos de enriquecimentos ambientais utilizados no experimento.

\begin{tabular}{|c|c|}
\hline Enriquecimento & Descrição \\
\hline Social & $\begin{array}{llr}\text { Contato } & \text { físico } & \text { com } \\
\text { outros animais } & \text { e } \\
\text { tratador; } & \end{array}$ \\
\hline Cognitivo & $\begin{array}{l}\text { Pneus suspensos na } \\
\text { baia e toras de } \\
\text { madeira para } \\
\text { escalagem; }\end{array}$ \\
\hline Físico & $\begin{array}{l}\text { Brinquedos } \\
\text { confeccionados com } \\
\text { garrafa pet; }\end{array}$ \\
\hline Sensorial & $\begin{array}{l}\text { Músicas (clássica, } \\
\text { erudita, sacra e } \\
\text { popular instrumental), } \\
\text { brinquedos de pet e } \\
\text { escovação. }\end{array}$ \\
\hline Nutricional & $\begin{array}{l}\text { "Sorvete" } \\
\text { com }\end{array} \begin{array}{r}\text { argarado } \\
\text { concentrado e capim } \\
\text { elefante) e varal de } \\
\text { folhagens } \\
\text { elefante). }\end{array}$ \\
\hline
\end{tabular}

Durante o reestabelecimento da hierarquia social, foram observados os padrões comportamentais sociais (agonísticos) e anormais. Diariamente foram realizadas três horas de observação por turno, sendo $1 \mathrm{~h} 30 \mathrm{~min}$ na baia controle (Trat 1) e 1h30min na baia de enriquecimento (Trat 2), perfazendo um total de $144 \mathrm{~h}$, sendo $72 \mathrm{~h}$ por tratamento.

Os comportamentos sociais agonísticos e anormais foram analisados por estatística descritiva. A comparação das médias da frequência dos comportamentos e do peso dos animais entre os tratamentos foi feita utilizandose o Proc GLM (SAS, 2008) e as médias foram comparadas pelo Teste $t$ (2008). 


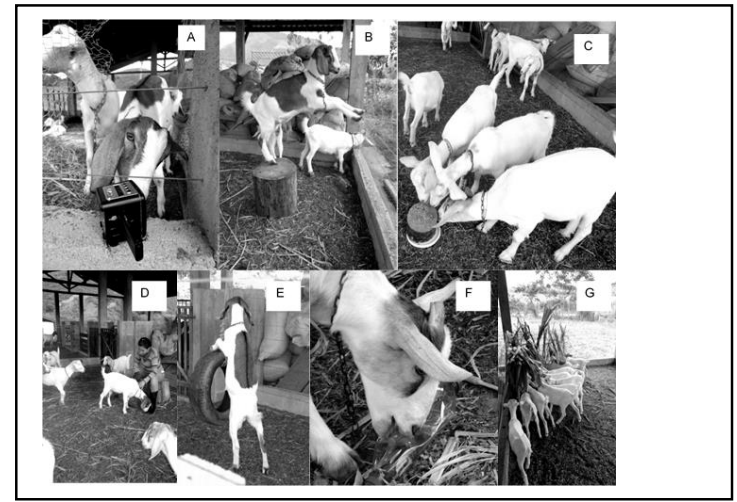

Figura 1 - Enriquecimentos ambientais ofertados às cabras. A) Música, B) Tora de eucalipto, C) Sorvete de capim e concentrado, D) Escovão, E) Pneu amarrado em corda, F) Brinquedo de garrafa pet e G) Varal de forragens feito com capim elefante amarrado em corda.

\section{RESULTADOS E DISCUSSÃO}

Os comportamentos sociais agonísticos identificados entre as cabras foram: dar cabeçada, ameaçar, morder, empurrar, tirar do cocho, perseguir e se isolar (Tabela 2).

Quanto ao padrão de comportamentos anormais, os mais observados foram autodestruição, apetite depravado e reatividade anormal (Tabela 3).

Os resultados encontrados neste trabalho estão de acordo com Paranhos da Costa (1988), que defende que caprinos confinados em ambientes empobrecidos em estímulos desenvolvem diversos comportamentos anormais, tais como estereotipias, autodestruição, falhas comportamentais, apetite depravado, reatividade anormal e autodestruição.

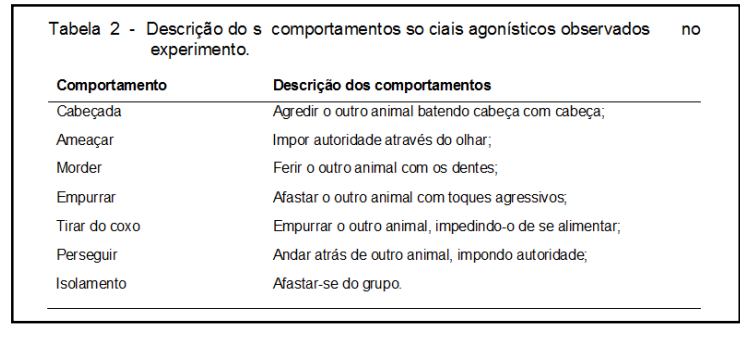

Tabela 3 - Descrição dos comportamentos anormais observados no estudo.

\begin{tabular}{|c|c|}
\hline Comportamento & $\begin{array}{l}\text { Descrição dos } \\
\text { comportamentos }\end{array}$ \\
\hline Autodestruição & $\begin{array}{l}\text { Comportamentos } \\
\text { de automutilação, } \\
\text { tais como, } \\
\text { morder seu } \\
\text { próprio pelo e/ou } \\
\text { partes do próprio } \\
\text { corpo; }\end{array}$ \\
\hline $\begin{array}{l}\text { Apetite } \\
\text { depravado }\end{array}$ & $\begin{array}{l}\text { Ingestão de } \\
\text { madeira, cama, } \\
\text { terra, fezes, além } \\
\text { da própria dieta } \\
\text { (hiperfagia) e } \\
\text { morder baia; }\end{array}$ \\
\hline $\begin{array}{l}\text { Reatividade } \\
\text { anormal }\end{array}$ & $\begin{array}{l}\text { Apatia, } \\
\text { inatividade } \\
\text { prolongada, } \\
\text { hiperatividade, } \\
\text { reações de } \\
\text { pânico. }\end{array}$ \\
\hline
\end{tabular}

Ao comparar as médias dos comportamentos sociais agonísticos entre os tratamentos (Tabela 4), verificou-se que na baia de enriquecimento ambiental (Trat 1) esses comportamentos foram menos frequentes em relação à baia controle (Trat 2).

Dentre os comportamentos agonístico observa-se que 0 mais 
frequente foi dar cabeçada, evidenciando este comportamento como o característico dos caprinos.

Comportamentos agonísticos são importantes para o estabelecimento da hierarquia social (Barroso et. al., 2000). No entanto alguns comportamentos agonísticos, principalmente os relacionados à obtenção de alimentos, podem estressar o animal, ocasionando a apatia no confinamento (Estevez et. al., 2007).

Barroso et. al. (2000), comparando taxas de agressão entre diferentes sistemas de produção de caprinos, verificou que, no sistema intensivo, a taxa de agressão física era maior em comparação com o semiextensivo, por este oferecer uma menor área, levando ao aumento da competição no grupo. No presente estudo, com os resultados positivos do uso de enriquecimentos ambientais na diminuição dos comportamentos relacionados à agressividade, pode-se confirmar que esta técnica foi eficiente na construção de um ambiente mais próximo do natural.

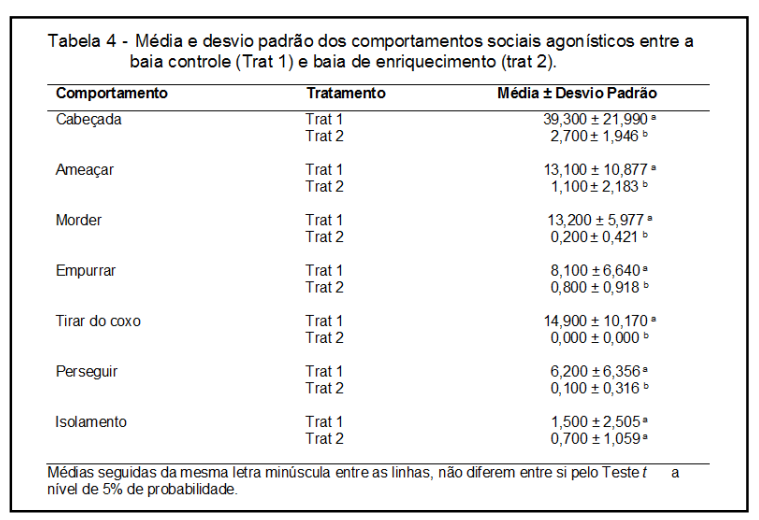

Em relação aos comportamentos anormais, verificou-se nos animais tratados com enriquecimentos ambientais (Trat 2), uma redução significativa na frequência dos comportamentos de autodestruição e apetite depravado (Tabela 5). As médias do comportamento de reatividade anormal não diferiram, sendo cabível ressaltar que este comportamento não foi observado no Trat 2.

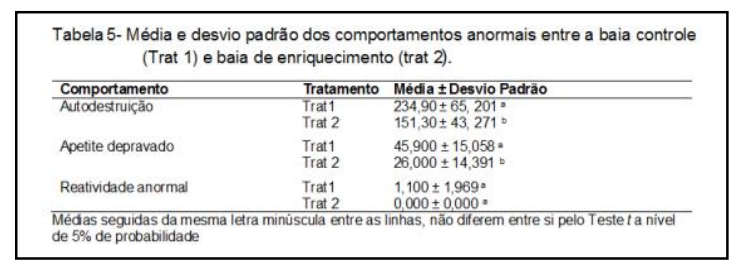

Os resultados representam a relevância dos enriquecimentos para a redução nos comportamentos anormais, no sistema intensivo de produção, que são considerados indicadores de baixo bem-estar. Segundo Wilson (1982), os enriquecimentos ambientais são utilizados para o aumento do bem-estar e o indicativo da eficácia dessa técnica é a redução de comportamentos anormais, como os comportamentos autodestrutivos e o desenvolvimento de atividades típicas da espécie (Novak e Suomi, 1988).

Os resultados do presente trabalho estão de acordo com os observados por Almeida et al. (2008) que discorre sobre o fato de que após a inserção de enriquecimentos ambientais em locais de confinamento, ocorrerem mudanças no comportamento, pois esta prática, que torna o ambiente mais estimulante, auxilia no processo contrário a apatia de cativeiro, como demonstrado em estudos desenvolvidos com primatas.

Em relação ao peso dos animais, verificou-se que as médias não foram diferentes entre os tratamentos. Na baia controle (Trat 1 ) a média inicial foi 23,200 $\pm 2,658$ e a média final foi $24,300 \pm 2,668$. $\mathrm{Na}$ baia de enriquecimento (Trat 2) a média inicial foi $20,700 \pm 3,056$ e a média final foi 20,100 $\pm 7,694$ (Tabela 6).

$\mathrm{O}$ resultado pode ser explicado pelo fato de que os animais da baia controle (Trat 1) permaneciam apáticos por horas, ou seja, não ganharam peso significativo, pois estavam estressados. 
Contudo, em função da apatia causada pelo confinamento, não perderam peso. Entretanto, os animais da baia de enriquecimento (Trat 2) movimentavamse grande parte do tempo, a fim de interagir com os enriquecimentos ambientais, o que pode ter ocasionado perda do peso e/ou o decréscimo do peso pode ter sido o resultado de um estresse inicial em função da troca de ambiente. Contudo, acredita-se que num prazo maior esses resultados possam apresentar-se mais eficientes para a produção. O que não se tem dúvida, é que o incremento dos enriquecimentos ambientais, como tática de manejo, melhorou efetivamente a qualidade de vida das cabras, podendo ser indicado o seu uso aos produtores, em função da eficiência e do baixo custo de implantação da técnica.

\section{CONCLUSÃO}

O uso de enriquecimentos ambientais auxiliou na redução de comportamentos sociais agonísticos e anormais das cabras mestiças (Saanen $\mathrm{x}$ Boer) em sistema de confinamento.

\section{REFERÊNCIAS}

ALMEIDA, A.M.R.; MARGARIDO, T.C.C.; FILHO, E.L.A. Influência do enriquecimento ambiental no comportamento de primatas do gênero Ateles em cativeiro. Arquivo de Ciência Veterinária e Zoologia da Unipar, Umuarama, v. 11, n. 2, p. 97102, 2008.

BARROSO, F.G.; ALADOS, C.L.; BOZA, J. Social hierarchy in the domestic goat: effect on food habits and production. Applied Animal Behaviour Science, v. 69, p. 35-53, 2000.

BURN, C.C.; DENNISON, T.L.; WHAY, H.R. Relationships between behaviour and health in working horses, donkeys, and mules in developing countries. Applied Animal Behaviour Science, v. 126, p. 109-118, 2010.
CAMPOS, J. A.; TINÔCO, I. de F.F.; SILVA, F.F. et al. Enriquecimento ambiental para leitões na fase de creche advindos de desmame aos 21 e 28 dia. Revista Brasileira de Ciências Agrárias, v.5, n.2, p. 272-278, 2010.

ESTEVEZ, I.; ANDERSEN, I.L.; NEVDAL, E. Group size, density and social dynamics in farm animals.In. . Applied Animal Behaviour Science, v. 103, p. 185-204, 2007.

HAMMERSCHMIDT, J. Desenvolvimento e aplicação de perícia em bem-estar animal. Dissertação (Mestrado em Ciências Agrárias) Universidade Federal do Paraná, 2010.

MACHADO FILHO, L.C.P.; HOTZEL, M.J. Bemestar dos suínos. In: Seminário Internacional de Suinocultura, 5., 2000, São Paulo-SP. Anais. Concórdia: Embrapa Suínos e Aves, 2000. p. 7083.

MALAFAIA, P.; BARBOSA, J.D.; TOKARNIA, C.H. Distúrbios comportamentais em ruminantes não associados a doenças: origem, significado e importância. Pesquisa Veterinária Brasileira, v. 31, n. 9, p. 781-790, set. 2011.

MENDES, R.C.; FARIA, H.A.de; SALVIO, G.M.M. Enriquecimento ambiental para leitões na fase de creche no IF Sudeste MG-campus Barbacena. In: SIMPÓSIO DE PESQUISA E INOVAÇÃO, 2.; SEMINÁRIO DE INICIAÇÃO CIENTÍFICA,1., Anais... Barbacena: IF Sudeste MG, p.22-24, 2011.

NOVAK M.A.; SUOMI, S. Psychological wellbeing of primates in captivity. Am Psychol, v.43, p.765-773, 1988.

PARANHOS DA COSTA, M.J.R., ANDRIOLO, A. Amamentação e Alo-amamentação em Búfalos (Bubalus bubalis). In: M. J. R. PARANHOS DA COSTA; CROMBERG V. U. (editores). Comportamento materno em mamíferos: bases teóricas e aplicações aos ruminantes domésticos, Ribeirão Preto - SP, p. 247-261, 1998.

PIZZUTO, C.S.; SGAI, M.G.F.G.; GUIMARÃES, M.A.B.V. O enriquecimento ambiental como ferramenta para melhorar a reprodução e o bemestar de animais cativos. Revista Brasileira de Reprodução Animal, Belo Horizonte, v.33, n. 3, p.129-138, 2009.

RIBEIRO, V.L.; BATISTA, A.M.V.; CARVALHO, F.F.R. et al. Seletividade e Composição da dieta ingerida por caprinos recebendo alimentação à vontade e restrita. Revista Brasileira de Ciências Agrárias, Recife, v.4, n.1, p.91-94, 2009.

SGAI, M.G.F.G. Avaliação da influência das técnicas de enriquecimento ambiental nos parâmetros endócrinos e comportamentais de 
Callithrix penicillata (sagui-de-tufospretos) mantidos em estabilidade social e isolados. 2007. p.113. Dissertação (Mestrado em Reprodução Animal) - Universidade de São Paulo, São Paulo, 2007.

SILVA, C.M. Comportamento e descrição do etograma de três grupos genéticos de caprinos confinados através do monitoramento de imagens. 2011, 102f. Dissertação (Mestrado em Zootecnia) - Universidade Federal da Paraíba, 2011.

WILSON S.F. Environmental influences on the activity of captive apes. Zoo Biol, v.1, p.201-209, 1982.

YEATES, J. W. Maximising canine welfare in veterinary practice and research: a review. The Veterinary Journal, v. 2, p. 271-278, 2011.

ZEDER M.A, Hesse B. The initial domestication of goats (Capra hircus) in the Zagros mountains 10,000 years ago. Science, v.287, p. 2254-2257, 2000. 